\title{
Continuous Separation of Colloidal Particles using Dielectrophoresis.
}

\begin{abstract}
Dielectrophoresis is the movement of particles in nonuniform electric fields and has been of interest for application to manipulation and separation at and below the microscale. This technique has the advantages of being noninvasive, nondestructive, and noncontact, with the movement of particle achieved by means of electric fields generated by miniaturized electrodes and microfluidic systems. Although the majority of applications have been above the microscale, there is increasing interest in application to colloidal particles around a micron and smaller. This paper begins with a review of colloidal and nanoscale dielectrophoresis with specific attention paid to separation applications. An innovative design of integrated microelectrode array and its application to flow-through, continuous separation of colloidal particles is then presented. The details of the angled chevron microelectrode array and the test microfluidic system are then discussed. The variation in device operation with applied signal voltage is presented and discussed in terms of separation efficiency, demonstrating $99.9 \%$ separation of a mixture of colloidal latex spheres.
\end{abstract}

Keyword: Colloidal; Dielectrophoresis; Separation 